\title{
Structural Integrity of Flat Silicon Panels for Nanosatellites - Modeling and Testing
}

\author{
Hugo Nguyen ${ }^{*}$, Robert Thorslund ${ }^{\dagger}$, Greger Thornell ${ }^{\ddagger}$, Johan Köhler ${ }^{\S}$, Lars Stenmark ${ }^{* *}$ \\ The Ångström Space Technology Centre, Department of Engineering Sciences, Uppsala University, Sweden
}

\begin{abstract}
To utilize the high mass fraction of silicon material in a nanosatellite based on microelectro-mechanical systems, part of the structural function has been assigned to the flat silicon stacks embracing these systems. Three modules for destructive testing in bending, warping and shearing cases were built with $68 \times 68 \times 1 \mathrm{~mm}$ silicon stacks bonded in aluminium frames by in-situ casting of silicone rubber. The rubber served as the deformation zone between the stiff and brittle silicon stacks and their weaker and ductile aluminium frames. A special test module of the same size was built with strain gauges of Nichrome (thin film deposited directly on the surface of the silicon stack). Elastic deformation tests on this as well as simulations using finite element analysis were performed for bending, warping and shearing loads of up to 80, 40 and $99 \mathrm{~N}$, respectively. The test module was disassembled after the test series and examined. The actual thickness of the rubber was measured and entered into the model for simulation. The correlation between simulations and experimental measurements was good with deviation of about $30 \%$. The results show that the rubber works well as a mechanical interface. Its thickness influences the stress in the silicon stack significantly. The silicon stack stiffens the module by a factor of 46 and lowers the stress in its frame 24 times in shearing mode, which is the most relevant loading case for the satellite framework. Thus, the concept of using flat silicon panels as structural elements is fully feasible.
\end{abstract}

\footnotetext{
* Ph.D. student, The Ångström Space Technology Centre, Uppsala University, Box 534, SE-751 21 Uppsala, Sweden.

† Research Engineer, The Ångström Space Technology Centre, Uppsala University, Box 534, SE-751 21 Uppsala, Sweden.

${ }^{\ddagger}$ Ph.D., The Ångström Space Technology Centre, Uppsala University, Box 534, SE-751 21 Uppsala, Sweden.

$\S$ Ph.D., The Ångström Space Technology Centre, Uppsala University, Box 534, SE-751 21 Uppsala, Sweden. Member AIAA.

${ }^{* *}$ Professor, The Ångström Space Technology Centre, Uppsala University, Box 534, SE-751 21 Uppsala, Sweden.
} 


\section{Nomenclature}

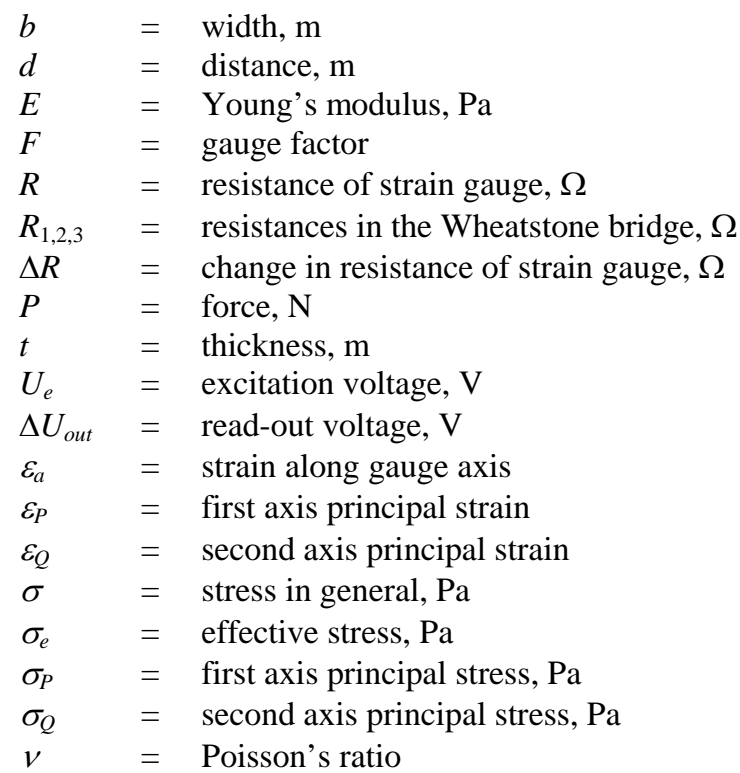

\section{Introduction}

On the way toward implementation of the nanosatellite NanoSpace-1 (NS1), ${ }^{1-9}$ based on Micro-ElectroMechanical System (MEMS) technology, a rigid, yet light-weight, selfsupported aluminium framwork design has been developed, Fig. 1a. Hence, this is a satellite body not divided into the traditional primary and secondary structures. Microsystems for this spacecraft are generally based on single crystalline silicon wafers and constitute a total mass of $1.7 \mathrm{~kg}$, whereas the mass budget the framework is $1.0 \mathrm{~kg}{ }^{9}$

An aluminium frame of fixed dimensions, Fig. 1b, has been adopted as a mounting standard to facilitate microsystems modularity for NS1 and its equivalents. The flat silicon-based microsystems are referred to as Multifunctional MicroSystem (MMS) modules or panels, because they have a number of functions, such as thermal management and radio transmission to fulfil. ${ }^{5}$ This concept improves the panel's total system performance per weight significantly in comparison with for example multifunctional structure or reconfigurable distributed architecture. ${ }^{9}$ The aluminium frame does not have any bracing or backing as the backside of the modules requires free passage for fluidic, electric and thermal connection. Similarly, the often functional front surface faces outer space from a defined distance to the front plane of the frame.

To accomplish a long technology lifetime the structural interface to the panel shall interfere as little as possible with the specific functions of the panel, but still allow for it to contribute to the structural integrity of the spacecraft. Furthermore, all panels shall facilitate assembly and disassembly.

In this concept, single crystalline silicon wafers embracing microsystems are bonded together, forming a stack mechanically rigid and strong enough to fulfil the load-carrying function. The thin-walled aluminium frames alone are not sufficiently strong. Since the aluminium frames and silicon stacks have very different mechanical and thermal properties, the direct contact between them could easily lead to mechanical failure during launch or in a fluctuating environment. Therefore, a thin silicone rubber interface is in-situ cured between the two materials, serving as a bonding element, a thermal conductor, and a deformation zone between them, Fig. 2.

The load carrying capability of the MMS module is investigated in this work through testing and modeling. Finite Element Analysis (FEA) is used for a feasibility study, sensitivity analysis, and to assess the reinforcement effect of the silicon stack on the aluminium frame. A special test module is manufactured and tested for strain versus load in three different cases, namely bending, warping, and shearing. In addition, three simpler modules were used in corresponding but destructive tests to obtain the ultimate strength of the module.

The primary objective of this paper is to show that the strength of the module is sufficient for spacecraft application. The second objective is to demonstrate that the rubber interfacing method is appropriate for flat silicon 
panels. Another goal is to accomplish appropriate modeling in an affordable personal computer environment for the design evaluation, which is an original requirement of this work.

Since all MEMS of the spacecraft are expected to be inactive during launch, their specific functions are not considered in this work. Rather, this work should be treated as the first step in the investigation of using flat silicon panels as structural elements.

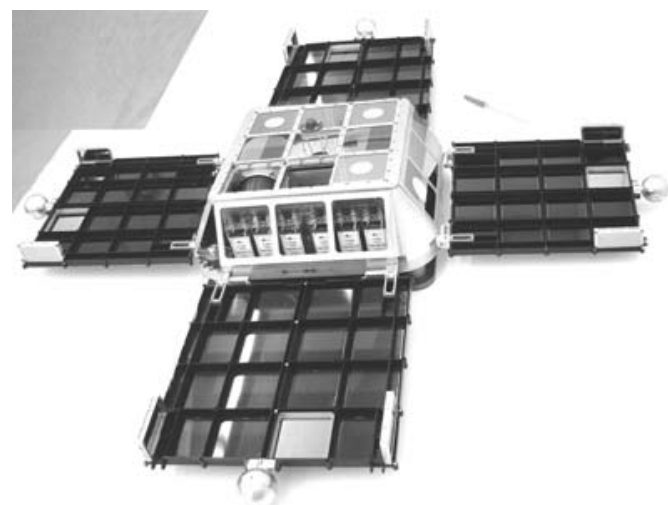

(a)

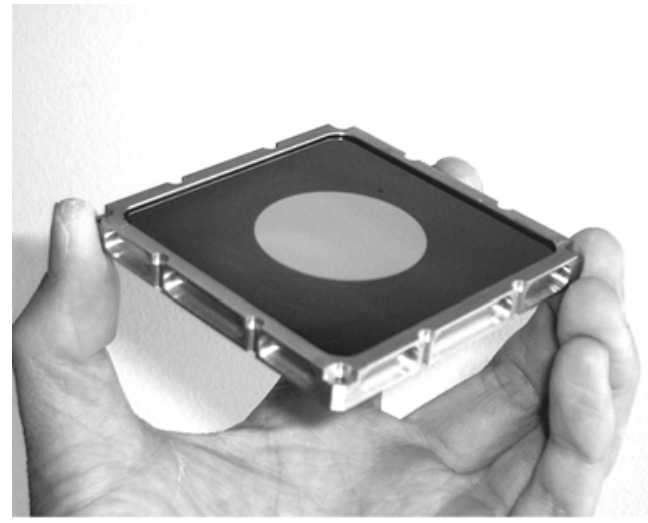

(b)

Fig. 1 Model of the nanosatellite NanoSpace-1, (a), and a multifunctional microsystem module, (b).

\section{Test module and test set-up}

Because the MMS panels currently consist of at least two wafers, the test module comprises a two-wafer stack (fusion bonding: $\mathrm{HNO}_{3}$-activation, anneal $1050^{\circ} \mathrm{C}$ for 18 hours) held in a standardized aluminium frame by in-situ cured rubber, Fig. 2. The frame has the outer dimensions of $74.60 \times 74.60 \times 7.50 \mathrm{~mm}$, whereas the silicon stack is $68 \times 68 \times 1.050 \mathrm{~mm}$. The total mass of this module is 22.635 gram.

A $150 \mathrm{~mm}$ thick layer of Nichrome was deposited on the silicon wafer by evaporation, and patterned by standard UV-lithography ${ }^{18}$ to form strain gauges.

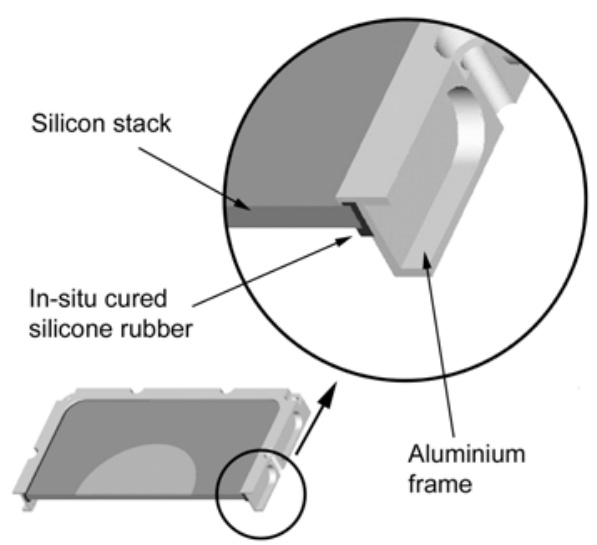

(a)

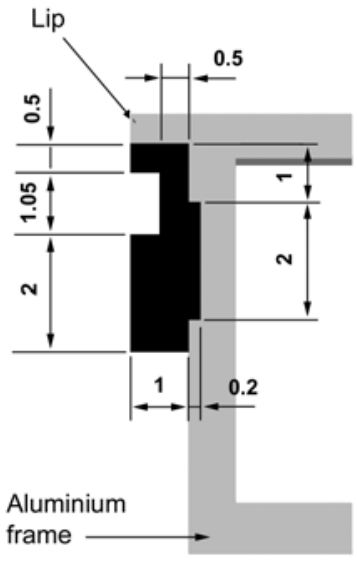

(b)

Fig. 2 General module with aluminium frame in cross section, (a), and the dimensions of the rubber in millimetres, (b).

The selected configuration for the strain gauges was the delta rosette, ${ }^{12}$ Fig. 3 (left). Two calibration beams and quality assessment structures were included in the design, Fig. 3 (right). 

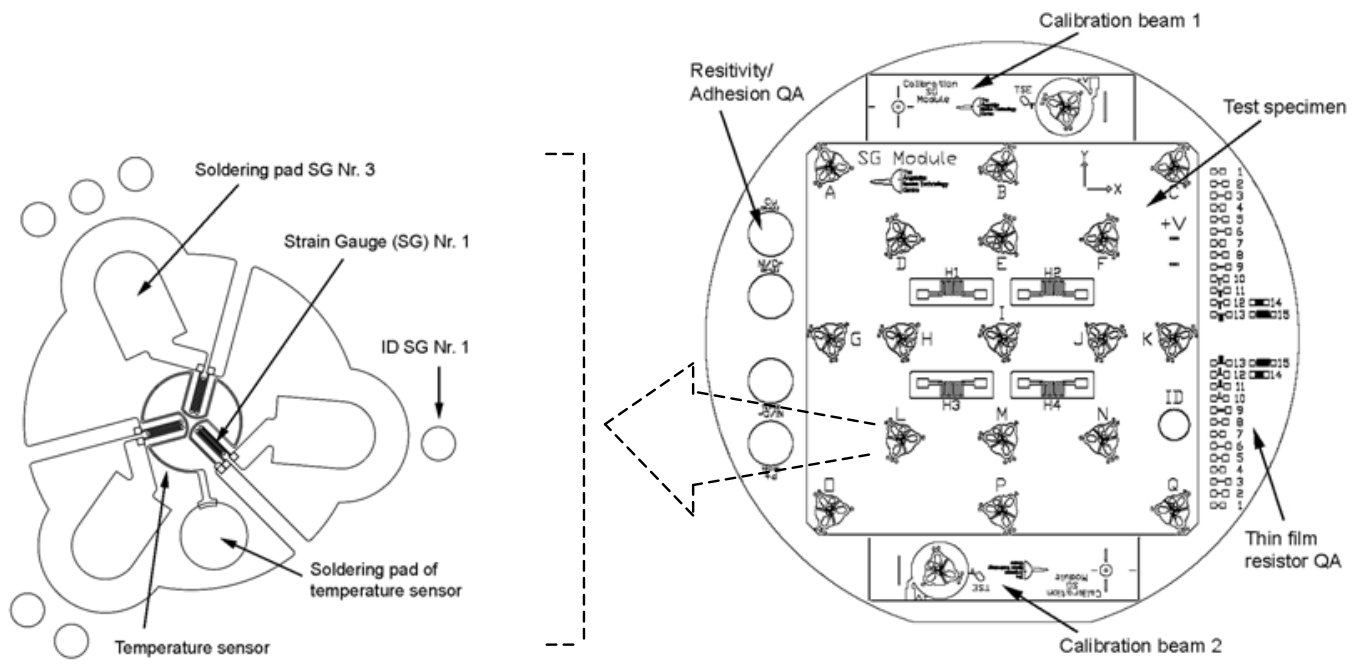

Fig. 3 Design of single delta rosette, (left), and test specimen, (right).

The square module with chamfered corners was diced out from the wafer and its corners further rounded off to a $0.8 \mathrm{~mm}$ radius for curvature by manual grinding. After placement in its frame the silicone rubber (Elastosil RT675, Wacker) was cast at room temperature with a special Teflon fixture ensuring alignment of the stack and a cavity for filling of rubber, Fig. 2. Curing was made at room temperature for 24 hours. After precision soldering lead-wires to the strain gauges, the test module was mounted rigidly to a test stand, and the lead-wires connected to an electronic data acquisition system, Fig. 4b. By relocation of the piezoelectric load cell, bending, warping, and shearing tests can be done in the same stand, Fig. 5.

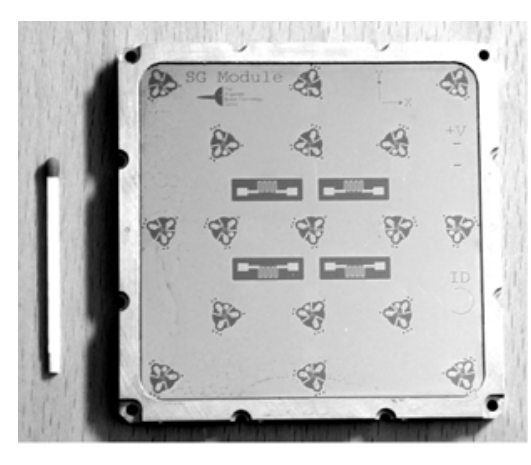

(a)

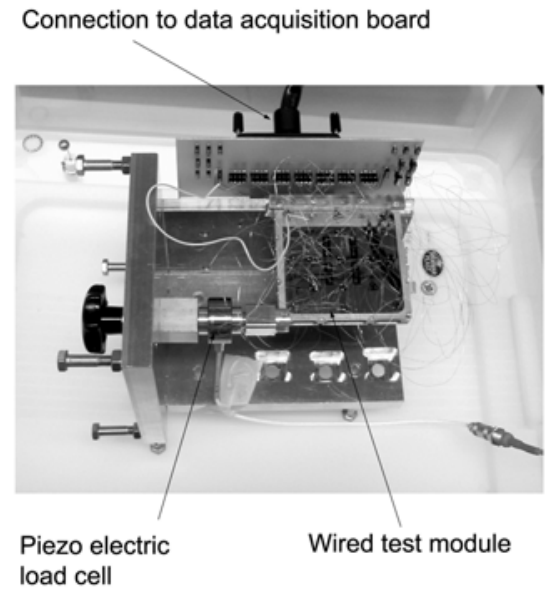

(b)

Fig. 4 Test module with strain gauges, (a), and test set-up with connection to data acquisition system, (b). 


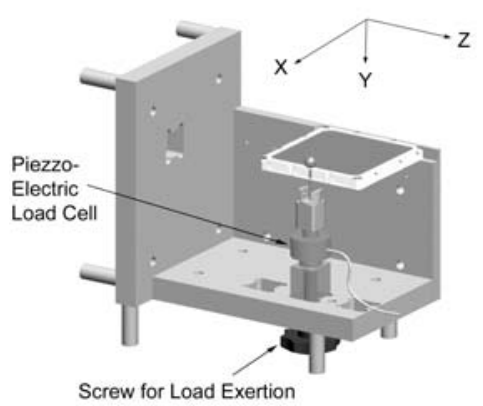

(a)

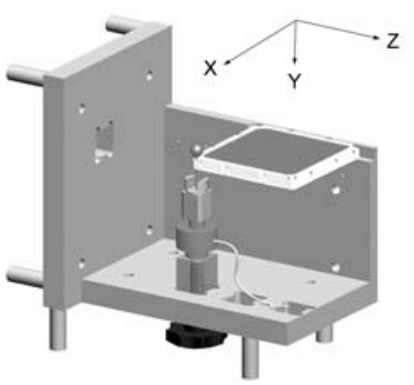

(b)

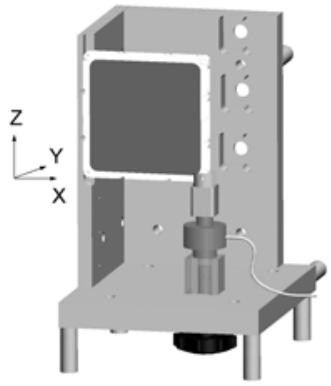

(c)

Fig. 5 Set-up configuration for bending, warping, and shearing tests (a, b, and c).

\section{Theory}

The unit change in resistance, $\Delta R / R$, of a strain gauge is proportional to the strain, $\varepsilon$, by the gauge factor, $F$, according to: ${ }^{13}$

$$
\frac{\Delta R}{R}=F \varepsilon
$$

The error due to the transverse sensitivity, that is the behaviour of the gauge in response to the strain perpendicular to the gauge axis, ${ }^{14}$ is zero for all strain gauges in the delta rosettes that are parallel to the calibration gauge. The reason is that in this work, the strain gauges were manufactured directly on the test module, and the gauge factor was measured on a beam diced out of the same silicon wafer as the test module.

The calibration cantilever was rigidly clamped to a stand and subjected to bending for calibration. Figure 6 shows the design principle for the calibration beam with gauge 1 as the calibration gauge, and gauge 2 (identical but orthogonal to gauge 1) for detection of the unwanted warping of the beam.

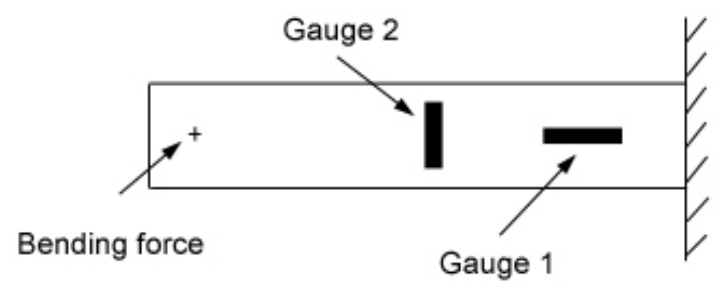

Fig. 6 Schematic top-view of calibration beam with bending force applied at free end and perpendicular to the main surface

The dimensions of the cross section of the beam, and the location of the gauges were governed by the design of the lithographic mask and the stack thickness. Ordinary beam theory provides the axial strain, $\varepsilon_{a}$, as:

$$
\varepsilon_{a}=\frac{6 P d}{E b t^{2}}
$$

where $P$ is the applied force, $d$ the beam length, $t$ the beam thickness, $b$ the beam width, and $E$ Young's modulus.

The axial strain of the gauge is in fact identical to $\varepsilon$ in Eq. (1) when it is used in calibration.

The thermal effect on the output of the strain gauges is low, since Nichrome has a low temperature coefficient of resistance (TCR). ${ }^{15} \mathrm{~A}$ commercial resistor of Nichrome can be obtained with a TCR of $2 \mathrm{ppm} /{ }^{\circ} \mathrm{C}$, and a stability of $\pm 0.1 \% \Delta \mathrm{R} / \mathrm{R}\left(1000\right.$ hours at $+125^{\circ} \mathrm{C}$ and $\left.125 \mathrm{~mW}\right) .{ }^{16}$ With all tests being conducted at $22^{\circ} \mathrm{C}$, a thermal compensation was not made here. 
To investigate the gauge factor, the read-outs from calibration tests were converted into the $\Delta R / R$. The formula for calculation of $\Delta R$ is specific to the electronic measurement system used. Figure 7 shows schematically the simplified circuit with a Wheastone bridge for one strain gauge used here. Thus, the change in resistance $\Delta R$ of the strain gauge can be expressed as:

$$
\Delta R=\frac{R_{1}}{\frac{\Delta U_{\text {out }}}{500 U_{e}}+\frac{R_{2}}{R_{3}+R_{2}}}-R_{1}-R
$$

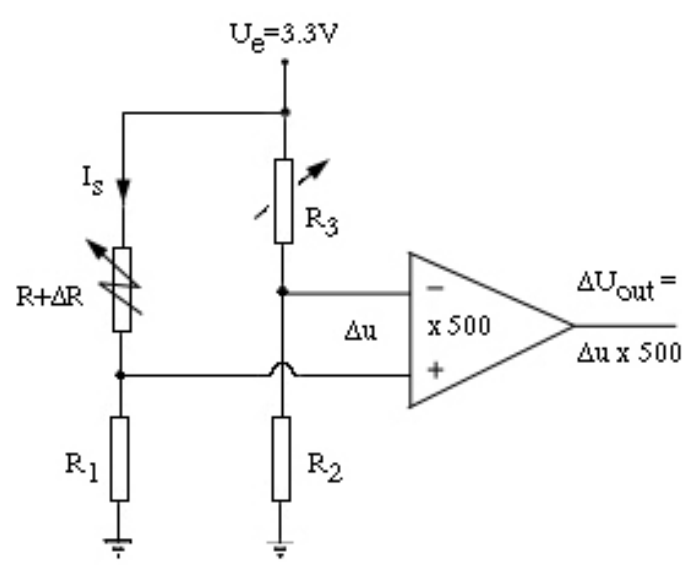

Fig. 7 Wheatstone bridge implemented in the electronic circuit, with $R+\Delta R$ representing the gauge and $R_{1}$ to $R_{3}$ balancing resistors

Read-outs from the multi-channel data acquisition system were converted into $\varepsilon_{1}, \varepsilon_{2}$, and $\varepsilon_{3}$ strains for each strain gauge delta rosette according to Eq. (1). From these values the principal strains $\varepsilon_{P}$ and $\varepsilon_{Q}$, and corresponding stresses $\sigma_{\mathrm{P}}$ and $\sigma_{\mathrm{Q}}$ could be calculated through: ${ }^{12}$

$$
\begin{gathered}
\varepsilon_{P, Q}=\frac{\varepsilon_{1}+\varepsilon_{2}+\varepsilon_{3}}{3} \pm \frac{\sqrt{2}}{3} \sqrt{\left(\varepsilon_{1}-\varepsilon_{2}\right)^{2}+\left(\varepsilon_{2}-\varepsilon_{3}\right)^{2}+\left(\varepsilon_{3}-\varepsilon_{1}\right)^{2}} \\
\sigma_{P}=\frac{E}{1-v^{2}}\left(\varepsilon_{P}+v \varepsilon_{Q}\right) \\
\sigma_{Q}=\frac{E}{1-v^{2}}\left(\varepsilon_{Q}+v \varepsilon_{P}\right)
\end{gathered}
$$

The commonly used von Mises stress was then obtained from:

$$
\sigma_{e}=\sqrt{{\sigma_{P}{ }^{2}+\sigma_{Q}{ }^{2}-\sigma_{P} \sigma_{Q}}}
$$

in case of the plane stress assumed here. 


\section{Experimentals and modeling}

\section{A. Calibration of strain gauges}

The resistance of the calibration gauges was measured at first without load, and then with dead weights of 43.44, 87.04, 125.54, 163.48, 201.52, 245.41, and 289.34 grams with a tree-minute-increment between the loadings.

A seven-digit ohmmeter monitored one gauge at a time. Nine calibration and five system drift tests were performed using the ohmmeter for 12 to 20 minutes each, with a sampling frequency of $2.5 \mathrm{~Hz}$. The multi-channel data acquisition system used in the experimental tests was used in parallel with the ohmmeter to check the compatibility. Computerized data sampling by means of LabView software was used in both cases to provide more precise average values of the signals from strain gauges. Eight calibration tests and seven system drift tests were performed using the multi-channel data acquisition system for 8 to 18 minutes each, with a sampling frequency of $0.33 \mathrm{MHz}$.

In the unloaded state $\Delta R$ is zero. Resistances $R_{1}$ and $R_{2}$ had been selected to be equal, and close to the values of $R$ and $R_{3}$ to gain high sensitivity of the Wheatstone bridge. When tuned to be in balance, by adjusting $R_{3}$ so that $R_{3}=R$, the $\Delta U_{\text {out }}$ was cancelled, see Eq. (3). From a practical perspective, the $R_{3}$ were adjusted so that $\Delta U_{\text {out }}$ approached zero (not necessarily exactly zero) before the calibration and test work, since an offset could be compensated for later in the treatment of the results.

\section{B. Measurement on test module}

Figure 8 shows schematically the three test cases: bending, warping, and shearing. Bending and warping forces were perpendicular to the test module, whereas the shearing force was directed along the edge of the module. The edge on the opposite side of the test module was rigidly clamped in all experiments.

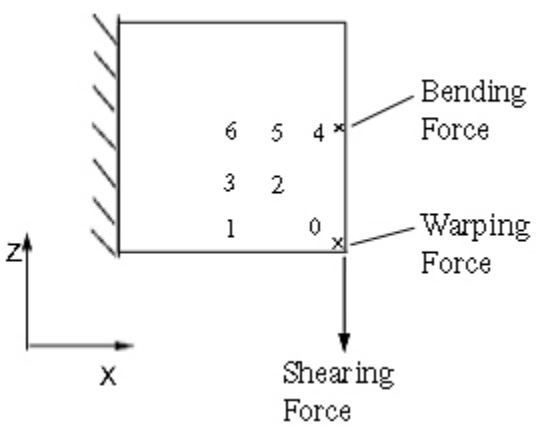

Fig. 8 Scheme for bending, warping, and shearing tests on the test module. Numbers 0 to 6 indicate the locations of the strain gauge delta rosettes. Module was clamped on the left side.

For symmetry reasons, only seven strain gauge rosettes in one quarter of the test module, Fig. 4a and 8, were used. The data acquisition system monitored signals from all 21 channels consecutively with a frequency of 0.33 $\mathrm{MHz}$ and a resolution of 12 bits. Loads were applied through the piezoelectric load cell (100Lb, 64.04.76), Fig. 5, in increasing order, starting at zero Newton, because of hysteresis in the load cell. In all tests the loads applied to the test module were registered by reading signals from the load cell via a separate data acquisition board. One minute was needed to set load, wait for steady state and sample data from all channels, with 501 readings each. Loads from $0 \mathrm{~N}$, with an equal load increment in between, were applied in each test series. Ten minutes pauses were taken between the series to allow the load cell to recover after unloading. The data acquisition software cancelled automatically the residual hysteresis effect in the load cell when a zero-load value was entered as the first load in a test series. Four test series in bending, three in warping and eight in shearing cases were performed. The total number of test series that the test module endured was, however, 16, 6 and 27 in the mentioned loading cases, respectively.

The position of the silicon stack in the frame makes the module unsymmetrical, Fig. 2, causing it to twist (lean out of the XZ-plane) during shear tests. It was impossible to prevent the test module from twisting without influencing the already weak signals from the strain gauges in this test case. Therefore, twisting was allowed in the shearing experiment. Due to the small deformation of the test module in this configuration, shearing tests were performed eight times; from 0 to $99 \mathrm{~N}$ with steps of $20 \mathrm{~N}$ in order to establish measurement consistency. 


\section{Destructive testing}

The destructive bending and shearing tests, Fig 9a and c, were performed in a more robust stand using a dynamometer, which registered the highest force, that is the load at fracture, in each test. The destructive test with warping load was performed in the stand for the measurement using the piezoelectric load cell for loading, Fig 9b. The destructive tests provided a baseline for decision on the load ranges applied in the experiments on the test module with the strain gauges.

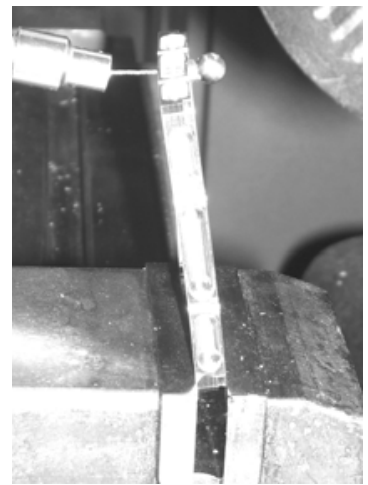

(a)

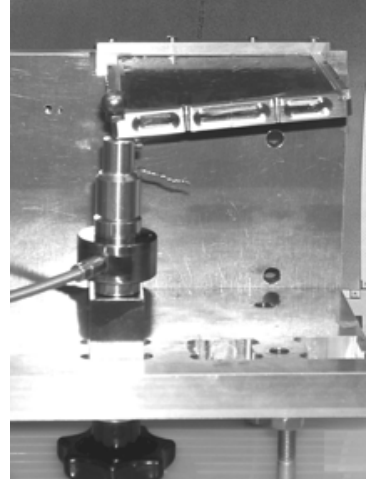

(b)

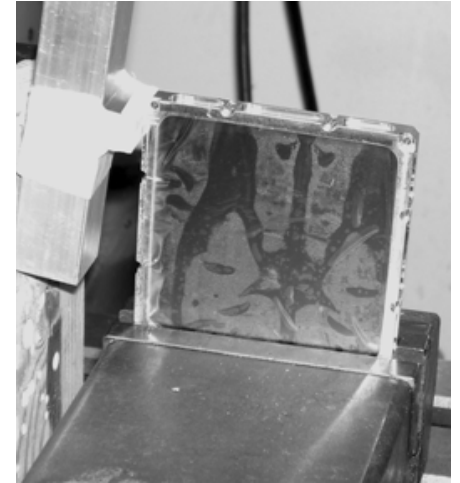

(c)

Fig. 9 Destructive testing in bending, warping, and shearing case (a, b, and c). Non of these panels here yet fractured. The modules have been equipped with a tape to take care of scattering.

\section{Characterization test of silicone rubber}

Silicone rubber in the form of a cylinder (26.9 $\mathrm{mm}$ in diameter, $63.2 \mathrm{~mm}$ in length) was compression tested (Instron 8516) to obtain the Young's modulus in different stress ranges for the FEA as this data was not available. The compression test proceeded until the sample fractured.

\section{E. Modeling}

The ProEngineer software used for design of the aluminium frame was also used for simulation of the three loading cases. The actual thickness of the rubber as obtained after disassembly of the test module was entered into the model. The silicon stack was modeled as linearly elastic with different Young's moduli, E, and Poisson's ratios, $v$, of silicon ${ }^{18}$ in each loading case: for bending $E=130 \mathrm{GPa}$ and $v=0.28$, warping $E=163 \mathrm{GPa}$ and $v=0.07$ and shearing $\mathrm{E}=168 \mathrm{GPa}$ and $v=0.064$, which correspond to main load-carrying directions of 0,38 and 45 degrees, respectively, from the $\mathrm{X}$-axis, Fig. 8. (The $\mathrm{X}, \mathrm{Y}$ and $\mathrm{Z}$ direction are all crystallographic [100] directions of the silicon wafer used.) These values were then corrected for the principal strain angle at each rosette location.

For the simulation, the $\mathrm{E}$ values of the rubber were iteratively determined by simulating the stress to which the rubber was subjected in each specific loading case. The E values were found at level between 10 and $12 \mathrm{MPa}$ corresponding to stresses between 0 and $1.78 \mathrm{MPa}$, and to strains between 0 and 0.10 due to the characterization test.

Simulations were performed for the same locations as in the experimental tests. Since the corrected results did not show a significant non-linearity, only three simulations were made for each loading case. Furthermore, although twisting could not be avoided during shearing experiments, a no-twist case was simulated to find out whether a large difference should be expected.

In the experimental tests strains at only a discrete number of locations on the surface of the silicon stack were measured. Therefore, FEA was employed as a complement in this work to estimate the reinforcement effect of the silicon stack on the aluminium frame. Simulations were made on aluminium frames both with and without the silicon stack, and for all loading cases. The reading were made at the most severe stress locations, see section E in chapter V, and the displacements taken at the loading points in the directions of the loads applied. One simulation for each loading case was sufficient. Also here, the highest loading forces, which correspond to the best signal-tonoise ratios in the experimental tests, that means 80, 40, and $99 \mathrm{~N}$ were selected for simulation of bending, warping, and shearing, respectively.

FEA was also employed to investigate the influence of the silicone rubber thickness on the stress in the silicon stack. The simulations were performed with the silicone rubber thickness decreased and increased with $50 \%$ from the designed thickness of $0.5 \mathrm{~mm}$. 


\section{Results}

\section{A. Calibration}

Figure 10 shows the characteristics of the two calibration gauges. The gauge factor was found to be 2.034. System drift affected the gauge factor by $1.28 \%$. The lower line is the characteristic of the transverse gauge on the beam divided by the negative value of Poisson's ratio $v=0.280$. It is presented in this way to avoid small negative values in the chart. Calculation of $\Delta R$ from calibration tests using the ohmmeter gave the same factor.

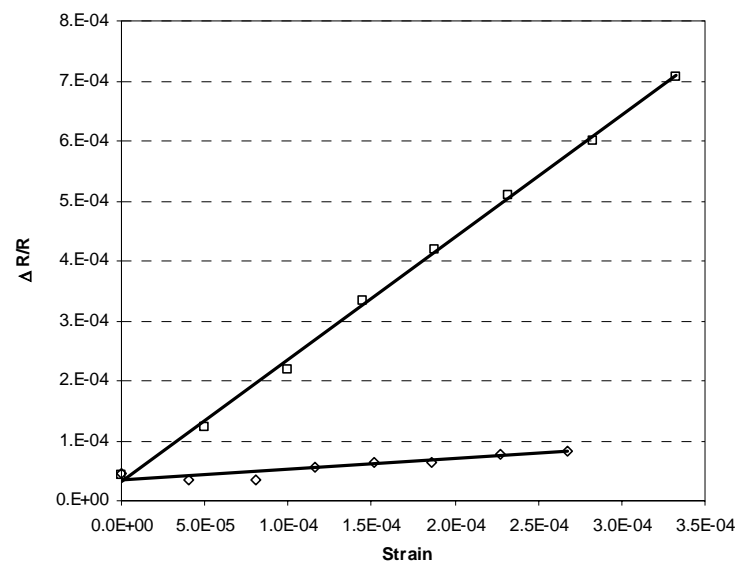

Fig. 10 Calibration curves for strain gauges.

By plotting the read-outs in voltage from calibration test versus axial strain calculated though Eq. (2), Fig. 11, the characteristics of the multi-channel data acquisition system could be obtained as $\Delta U_{\text {out }}=827.4 \cdot \varepsilon-0.0056$.

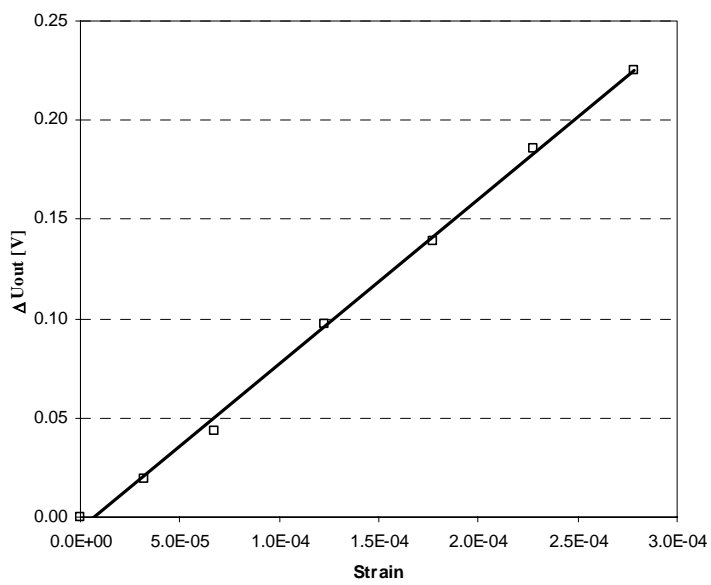

Fig. 11 Read-out in voltage versus strain for the multi-channel data acquisition system.

\section{B. Destructive tests}

The destructive bending, warping and shearing tests, Fig. 9, yielded 246, 239 and 865 N, respectively, with the module allowed to twist slightly in the shearing mode. The maximum displacement of the loading point and in the loading direction was measured to $7 \mathrm{~mm}$ in the warping case. The displacements in the two other cases could not measured properly due to the rougher set up, but was at least $7 \mathrm{~mm}$ in the bending and in order of a millimetre in the shearing case.

Fracture of the silicon stack in the bending case was a straight line parallel with the X-axis, Fig. 5a, initiated at the loading point. In the warping case the fractures was in the form of a few main cracks at about $38^{\circ}$ with respect to the X-axis. In the shearing case only a small piece at the corner opposite to the corner with applied load was damaged. The rest of the stack was intact. 
The aluminium frame was plastically deformed close to the clamped side in the bending case, whereas it was only elastically deformed in the other cases.

\section{Compression test of the silicone rubber and examination of the rubber in the test module}

The engineering stress of the rubber, as presented in Fig. 12, is calculated as the axial compression force divided by the original cross section area of the sample. In summary, the sample fractured at a stress of $5.4 \mathrm{MPa}$ and a strain of 0.32. From the graph in Fig. 12, Young's moduli of 10-12 and 49 MPa were chosen for modelling of stress of 01.78 and 4.26 MPa and above, respectively.

On disassembling the test module after a total number of 16 bending, 6 warping, and 27 shearing tests, microscopy showed that the rubber was intact.

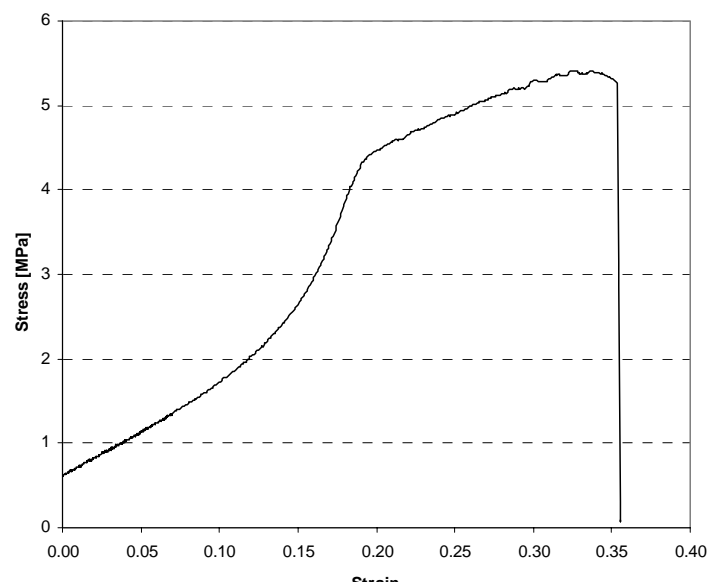

Fig. 12 Engineering stress versus strain for the silicone rubber.

\section{Test series and simulations}

The angles of principle strains calculated from the sampled data were used to determine $\mathrm{E}$ and $v$ of the silicon at each rosette location. ${ }^{17,18}$ These were needed for calculation of stresses, Eq. 5 and 6. Table 1 presents the average angles with respect to the $\mathrm{X}$-axis at each location, calculated from all test series for each loading case. The results from modeling were later corrected for the differences in $\mathrm{E}$ and $v$.

Delta rosette number 0 was not taken into further consideration due to its malfunctioning, as indicated already in Table 1, especially in the bending and shearing cases. Examination of this rosette using scanning electromicroscopy did not show any defects on gauges themselves, but poor soldering.

Table 1 Calculated angles in degrees of principle strains in bending, warping and shearing at each rosette location. (Rosette number 0 malfunctioned.)

\begin{tabular}{cccc}
\hline $\begin{array}{c}\text { Location } \\
\text { (Rosette) }\end{array}$ & Bending & Warping & Shearing \\
\hline 0 & 24 & 40 & 26 \\
1 & 6 & 32 & 36 \\
2 & 0 & 40 & 45 \\
3 & 0 & 35 & 40 \\
4 & 0 & 44 & 37 \\
5 & 0 & 41 & 45 \\
6 & 0 & 37 & 45 \\
\hline \hline
\end{tabular}

Figures 13 to 15 show the von Mises stress at the surface of the silicon stack in the three experimental loading cases and the corresponding modeling. 

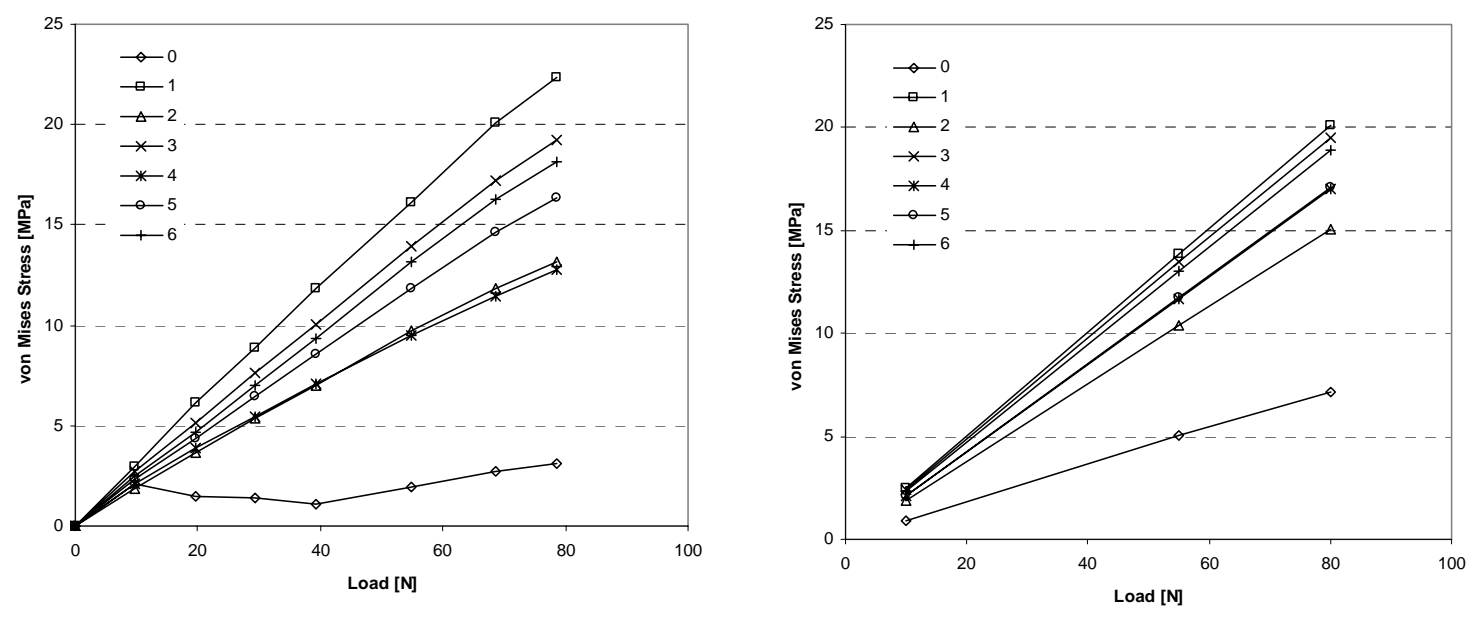

Fig. 13 Bending tests (left) and simulations (right). Numbers 0 to 6 denote different delta rosettes (Fig. 8) and corresponding locations on model.
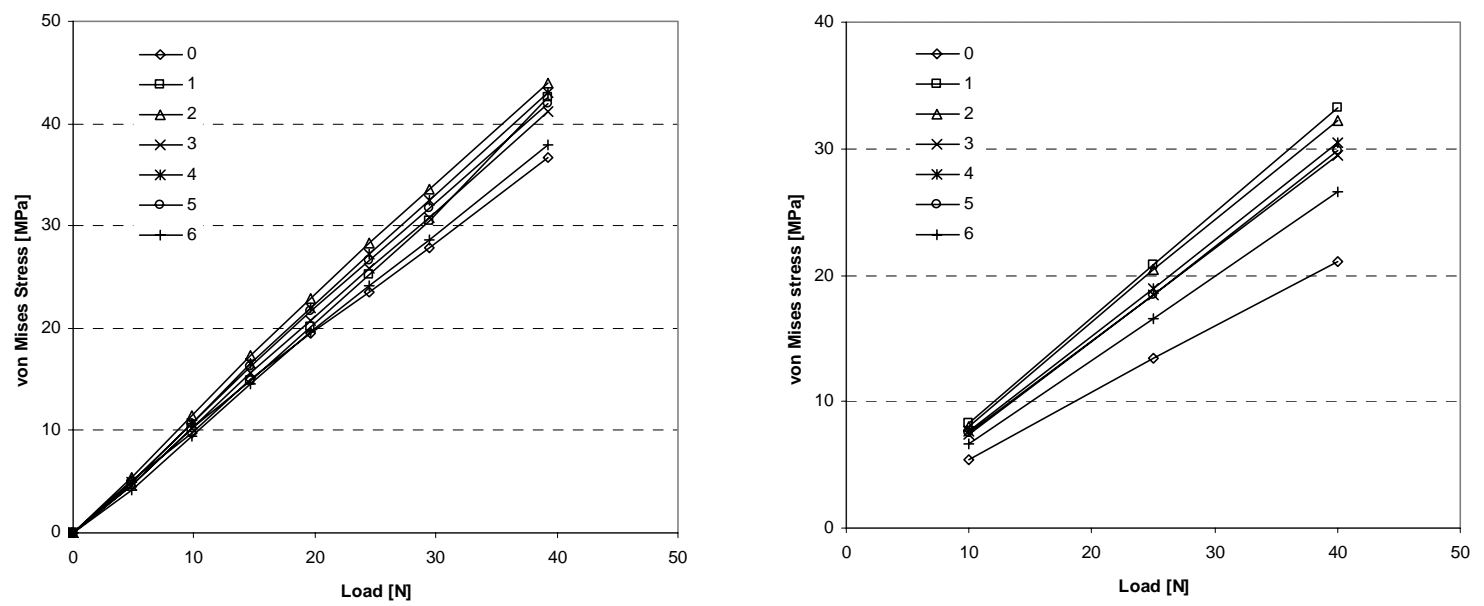

Fig. 14 Warping tests (left) and simulations (right). Numbers 0 to 6 denote different delta rosettes (Fig. 8) and corresponding locations on model.
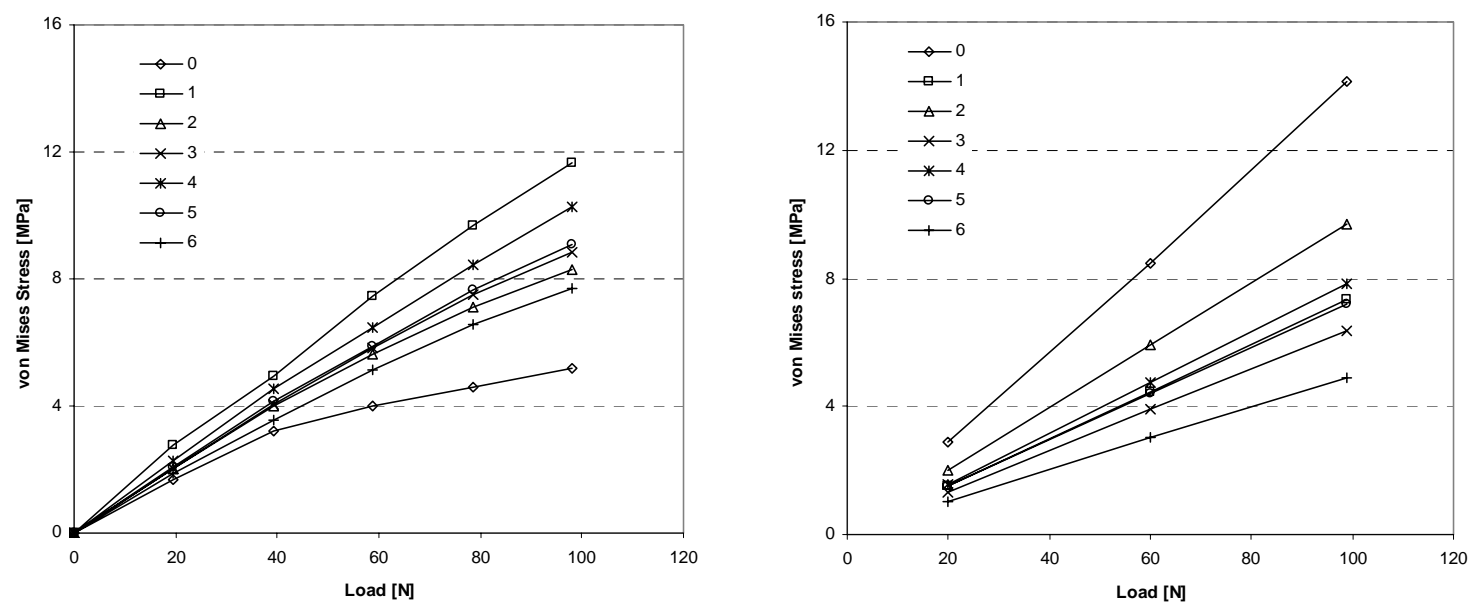

Fig. 15 Shearing-twist tests (left) and simulations (right). Numbers 0 to 6 denote different delta rosettes (Fig. 8) and corresponding locations on model. 
For comparison, the von Mises stresses from experiments and simulations with the highest applied loads are presented in Table 2, and the von Mises stress from simulations on shear-twist case and shear-no-twist case in Table 3.

Table 2 von Mises stresses on the silicon stack from experimental tests versus simulations at corresponding locations. Values in parentheses are the differences from the experimental tests in percent.

\begin{tabular}{ccccccc}
\hline $\begin{array}{c}\text { Location } \\
\text { (Rosette) }\end{array}$ & $\begin{array}{c}\text { Bending 80 N } \\
\text { [MPa] }\end{array}$ & $\begin{array}{c}\text { Warping 40 N } \\
\text { [MPa] }\end{array}$ & $\begin{array}{c}\text { Shearing-Twist 99 N } \\
\text { [MPa] }\end{array}$ \\
\hline 0 & $3.1 / 7.1 \quad(127 \%)$ & $37.5 / 21.1$ & $(-44 \%)$ & $5.2 / 14.1$ & $(169 \%)$ \\
1 & $22.8 / 20.1 \quad(-12 \%)$ & $43.4 / 33.2$ & $(23 \%)$ & $11.8 / 7.3$ & $(-38 \%)$ \\
2 & $13.4 / 15.1 \quad(12 \%)$ & $44.9 / 32.3$ & $(-28 \%)$ & $8.4 / 9.7$ & $(16 \%)$ \\
3 & $19.6 / 19.5 \quad(0 \%)$ & $41.9 / 29.4$ & $(-30 \%)$ & $8.9 / 6.4$ & $(-29 \%)$ \\
4 & $13.0 / 17.0 \quad(30 \%)$ & $44.0 / 30.5$ & $(-31 \%)$ & $10.4 / 7.8$ & $(-25 \%)$ \\
5 & $16.7 / 17.1 \quad(3 \%)$ & $42.7 / 29.8$ & $(-30 \%)$ & $9.2 / 7.2$ & $(-21 \%)$ \\
6 & $18.5 / 18.9$ & $(2 \%)$ & $38.6 / 26.6$ & $(-31 \%)$ & $7.8 / 4.9$ & $(-37 \%)$ \\
\hline \hline
\end{tabular}

Table 3 von Mises stresses on the silicon stack from simulation allowing and disallowing twist during shearing with $99 \mathrm{~N}$

\begin{tabular}{cccc}
\hline \hline $\begin{array}{c}\text { Location } \\
\text { (Rosette) }\end{array}$ & $\begin{array}{c}\text { Shearing-Twist } \\
\text { [MPa] }\end{array}$ & $\begin{array}{c}\text { Shearing-No-Twist } \\
\text { [MPa] }\end{array}$ & Ratio \\
\hline 0 & 14.1 & 5.3 & $\mathbf{2 . 7}$ \\
1 & 7.3 & 1.3 & $\mathbf{5 . 8}$ \\
2 & 9.7 & 0.8 & $\mathbf{1 2}$ \\
3 & 6.4 & 1.9 & $\mathbf{3 . 3}$ \\
4 & 7.8 & 1.6 & $\mathbf{5 . 0}$ \\
5 & 7.2 & 2.5 & $\mathbf{3 . 0}$ \\
6 & 4.9 & 3.3 & $\mathbf{1 . 5}$ \\
\hline \hline
\end{tabular}

Table 4 presents the maximum von Mises stress and the corresponding modulus for the rubber when subjected to the highest load in each case.

Table 4 Maximum von Mises stress in modeled rubber with highest applied load in all cases.

\begin{tabular}{lcc}
\hline \hline Test Case & $\begin{array}{c}\text { Highest von Mises } \\
\text { stress [MPa] }\end{array}$ & $\begin{array}{c}\text { Applicable Young's } \\
\text { module of rubber [MPa] }\end{array}$ \\
\hline Bending 80N & 1.75 & 12 \\
Warping 40N & 1.30 & 11 \\
Shearing-Twist 99N & 1.78 & 12 \\
Shearing-No-Twist 99N & 1.73 & 12 \\
\hline \hline
\end{tabular}

\section{E. Reinforcement factor}

Results from the simulations of the aluminium frame with and without the silicon stack are presented in Table 5. Readings were made at the most critical locations of the frame with respect to effective stress. That is on the surface of the thin lip of the aluminium frame side, Fig. 2, which is fixed to the stand at right angle at one end and carrying 
load at the other for the shearing and warping case. For the bending case, this is either one of those sides due to the symmetry. The location for reading the maximum stress is on the frame's inside at distance of $14 \mathrm{~mm}$ from the outer edge of the frame fixed in the stand for bending and warping cases, and 19 and $27 \mathrm{~mm}$ for shearing-twist and shearing-no-twist cases, respectively, Fig. 16. Displacements of the aluminium frame were read at the loading point and in the loading direction in each case.

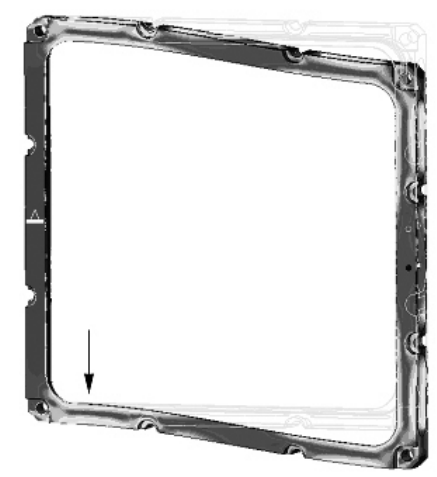

Fig. 16 Critical location of stress in the frame. The left side of the frame was rigidly clamped.

Table 5 Comparison of results from stress simulations on the aluminium frame with and without silicon stack

\begin{tabular}{lccc}
\hline \hline Test Case & $\begin{array}{c}\text { Frame with } \\
\text { silicon stack }\end{array}$ & $\begin{array}{c}\text { Frame without } \\
\text { silicon stack }\end{array}$ & Ratio \\
\hline a) von Mises stress [MPa] & & & \\
Bending 80 N & 190 & 200 & $\mathbf{1 . 0 5}$ \\
Warping 40 N & 170 & 190 & $\mathbf{1 . 1 2}$ \\
Shearing-Twist 99 N & 25 & 350 & $\mathbf{1 4}$ \\
Shearing-No-Twist 99 N & 17 & 407 & $\mathbf{2 4}$ \\
b) Displacement in loading direction [mm] & & \\
Bending 80 N & 1.18 & 1.30 & $\mathbf{1 . 0 9}$ \\
Warping 40 N & 0.97 & 1.15 & $\mathbf{1 . 1 8}$ \\
Shearing-Twist 99 N & 0.12 & 4.13 & $\mathbf{3 5}$ \\
Shearing-No-Twist 99 N & 0.08 & 3.84 & $\mathbf{4 6}$ \\
\hline \hline
\end{tabular}

F. Stress sensitivity with respect to thickness of the rubber

Figure 17 presents simulations performed with different thickness of the silicone rubber. Von Mises stresses from simulations with the rubber thickness of $0.5 \mathrm{~mm}$ were used as references.
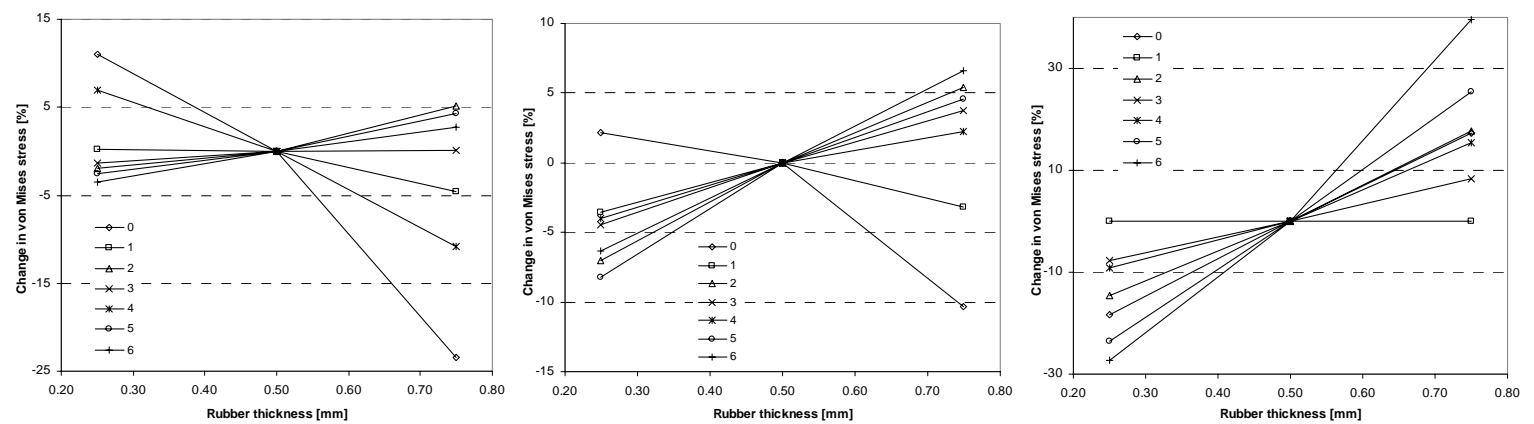

Fig. 17 Sensitivity of stress in the silicon stack in bending (left), warping (middle) and shearing case (right) with respect to rubber thickness. 


\section{Discussion}

From the results in general, but perhaps mostly from the reinforcement and sensitivity analysis, Table 5 and Fig. 17, the importance of the silicon stack for the stiffness of the module is evident. It could be argued that the aluminium frames of the framework could be strengthened in other ways - for instance by conventional cross bracing. For a relatively flat structure as the NS1 spacecraft, this is particularly true in case of shearing loads. However, the silicon stacks are vital parts of a microsystems-based nanosatellite, and this should be considered a way to make dual use of them and save mass. At the same time the stacks are given plenty of room for electric and fluidic interfacing to the interior of the vehicle, and the option of full exposure to space which is a prerequisite for modules housing many of the mission-specific instruments.

It has been shown how to account for the anisotropy of silicon (see section A below) which is important when the stacks consist of crystallographically aligned wafers. Future scenarios may involve wafers of a larger number bonded in a way permitting free orientation and hence a close to isotropic behaviour.

Also beyond the scope of this work is a full sensitivity analysis. The stack size adopted here has been chosen based on the maturity of the technology - and hence on what is efficient with respect to processing and cost. With an exception for the discussion on rubber (see section D below), scaling of the modules thickness and lateral size, as well as the dimensions and properties of the aluminium frame must be the subject of a separate treatise to which this work will hopefully serve as a guideline and source of inspiration.

\section{A. Material issues}

The silicon wafers used here were (100) oriented with the sides of the test module aligned to the $<100>$ directions. From the collected data the strains, $\varepsilon_{1}, \varepsilon_{2}$ and $\varepsilon_{3}$, for each delta rosette could be calculated, and thus the angle of the principle strains determined. This angle is needed to determine $\mathrm{E}$ and $v$ of the material at each location and load, Eq. 4 to 6.

The use of the main angle (in bending and shearing case), alternatively the averaged angle (in warping case) of principle strains in the modeling means that the silicon stack in the model was approximated with an isotropic material with $\mathrm{E}$ and $v$ dependant on the load carrying direction. The approximation was satisfactory entailing small correctable errors of up to $1.1 \%$ in a few locations, Table 1.

The iterative simulation to find proper $\mathrm{E}$ values for the rubber in each case to circumvent the non-linear modeling issue is applicable because the simulation is based a static load requiring only a specific point of the nonlinear behaviour of the material. For stress in the range of 0 to $1.78 \mathrm{MPa}$ the $\mathrm{E}$ values of the rubber changed from 10 to $12 \mathrm{MPa}$ in an almost linear part of the stress-strain curve of the material, Fig.12.

The simplification of the silicon to an isotropic material and the rubber to a linear one in the modeling as described above, the correlation between the simulation results and experimental results was in an acceptable range of about $30 \%$, Table 2 .

\section{B. Module testing and simulation reliability}

The upper line in Fig. 10 is typical for an uniaxial strain gauge with its gauge factor of 2.034 (2.008 after systemdrift compensation) being close to that of commercial Nichrome gauges. The lower line for the transverse gauge with an $\mathrm{R}^{2}$ value of 0.82 is governed by the drift and noise level in the electronics meaning that warping of the beam during bending could be neglected. Thus, the correctness of the calibration test is confirmed.

Although the loads at fracture were very high in all loading cases, the maximum loads applied to the test module with strain gauges were selected to 80, 40 and $99 \mathrm{~N}$, which are 3, 6 and 9 times lower, for bending, warping and shearing case, respectively. These loads were sufficiently high to generate good signals from the gauges, but low enough to avoid risk of damaging the gauges and the stand.

The bending tests produced good signals owing to the large deformations, and thus large strains. The poor correlation between the line for stress from delta rosette 0 in Fig 13 (left), compared with Fig 13 (right) is caused by the rosettes malfunctioning due to the poor soldering. Simulations for bending were performed with $\mathrm{E}$ and $v$ of the silicon corresponding to the [100] direction, whereas the angle at location 1 was calculated to $6^{\circ}$, Table 1 Table . Simulation with $\mathrm{E}$ and $v$ corresponding to this angle gave a roughly $1.1 \%$ higher stress at this location.

For location 4, the large difference of $30 \%$, Table 2, is believed to come from the set up where the steel wire used for transferring the bending load was somewhat too short and stiff perhaps creating a torque in negative $\mathrm{Z}$ direction at the load point close to rosette 4, Fig. 5a.

The warping tests produced a roughly two times larger stress in the test module although the applied loads were only up to half of those in the bending case, Fig. 14. The experimental and simulated stress differ roughly $30 \%$ for all locations. 
The shearing tests produced signals of much lower level than the two other cases, Fig 15, due to the smaller deformations of the test module in this configuration. Hence, the outputs from the gauges were not as strong despite higher applied loads. The highest stress in this case was about $12 \mathrm{MPa}$, whereas in the bending and warping case the maximum stress was two and almost four times higher, respectively. Also here, the line for stress at location of rosette 0 shows the rosette malfunction.

\section{Other results from simulation}

The so called shearing-no-twist case could not be experimentally tested due to the signal from the strain gauges being even weaker than that in the shearing-twist case. However, the case is relevant to be examined by modeling, as for a panel on a satellite's body such as that of NS1, Fig. 1a, twisting is likely to be limited. Comparison of the results from simulations shows that stress in the shearing-no-twist case is 1.5 to 12 times lower than in the shearingtwist case, Table 3.

In the concept described here, the rubber of only $0.5 \mathrm{~mm}$ in thickness has to transfer mechanical load between the frame and the panel, but also prevent them from making direct contact with each other, so that stress concentration could be avoided. Like many other elastomers, the silicone rubber is non-linearly elastic, making it increasingly stiff on compression. Thus, the silicone rubber is expected to work as a progressive cushion between the two materials. The iterative simulation converged towards an E of 10 to $12 \mathrm{MPa}$ for the rubber with loads up to 80, 40, and $99 \mathrm{~N}$ in bending, warping, and shearing, respectively, Table 4, whereas the E before plastic deformation of the rubber was found to be $49 \mathrm{MPa}$, Fig. 12. On examination after disassembling of the module the rubber was found to be intact, confirming that it was subjected to only moderate stresses. However, the load carrying capacity of the rubber might be higher than the sample in the characterization test, because in the test module the rubber is almost enclosed between the aluminium frame and the silicon stack.

\section{Stress dependence on the rubber thickness}

Generally, thinner rubber is desirable since it entails lower stress in the silicon stack. In the bending and warping cases, location 0, 1 and 4 should be monitored when the rubber thickness decreases due to the increase of the stress. Although the stress dependence on the rubber thickness, expressed in percent and with respect to the thickness of 0.5 $\mathrm{mm}$, is lower for bending and warping than for shearing, attention should be paid to the much higher stress level in the first two cases.

\section{Conclusion}

Both experimental tests and simulations show that the concept of using flat silicon panels to strengthen an aluminium framework of such as that employed by the nanosatellite Nanospace-1 is feasible.

Bending, warping and shearing were simulated and experimentally tested with point loads of up to 80, 40 and 99 Newtons, with resulting maximum stresses of 20, 33 and $14 \mathrm{MPa}$, respectively, in the silicon panel, and simulated stresses typically deviated from this with $30 \%$. Destructive tests in the three loading cases indicated fracture load 3, 6 and 9 times higher and fracture patterned in support of the direction of effective loading anticipated.

Shearing is the most relevant loading case for this spacecraft, since the overall framework using flat silicon panels as its walls, prevents the panels from large deformations in bending and warping mode. Simulation for shearing has shown that the silicon stack stiffens the aluminium frame 46 times with respect to displacement, and lowers its stress at the most critical location 24 times.

The concept of using silicone rubber as a mechanical interface between a stiff and brittle material like silicon being typical for micromechanical system, and a conventional metallic building element has also been demonstrated. Here, it works both as bonding element for a silicon stack in an aluminium frame, and as a deformation and stress distribution zone between the two materials which have very different mechanical and thermal properties. The rubber endured most deformation in the almost linear part of its stress-strain characteristic in the experiments. The highest stress it suffered was 1.78 MPa under the load of $99 \mathrm{~N}$ in shearing, whereas its yield and fracture stress were found to be 4.26 and $5.40 \mathrm{MPa}$, respectively. It found that thin rubber lowers the stress in the silicon panel, whereas thick rubber increases the stress.

\section{Acknowledgments}

The authors would like to express their gratitude to the European Space Agency for financial support under the Micro/Nano Satellite Technlogy project, of which this work is a part. A great thank is addressed to research engineer Sven-Erik Jansson, at The Swedish Institute of Space Physics, for his excellent help in building of measurement system, and to Anders Jansson at the Department of Solid Mechanics, and professor Ram Gupta at the Department 
of Material Science, Uppsala University, for their enthusiastic help and discussion on the theory part. Also, thank you Peter Nilsson Zandkarimi, Anders Eriksson and Johan Bejhed, former and present colleagues at The Ångström Space Technology Center for your kind assistance.

\section{References}

${ }^{1}$ Bruhn, F., Köhler, J., and Stenmark, L., ”Nanospace-1: The Impact of The First Swedish Nanosatellite in Spacecraft Architecture and Design,” Acta Astronautica, Vol. 53, Issue 4-10, August 2003, pp.633-643.

${ }^{2}$ Nguyen, H., Köhler, J., Stenmark, L., "The Merits of Cold Gas Micropropulsion in State-of-the-art Space Missions," The 53rd International Astronautical Congress (World Space Congress), Houston, Texas, 2002, pp IAC-02-S.2.07

${ }^{3}$ Nguyen, H., et.al., "Micropropulsion Systems Research and Manufacture in Sweden," 4th Round table of Micro- and Nanotechnologies for Space, proceedings, May 2003, Estec, Noordwijk, The Netherlands.

${ }^{4}$ Kratz, H., et.al., "Nanospacecraft Technology and its Predicted Consequences for the Space Community" IEEE, Transactions on Aerospace and Electronic Systems, Submitted paper, September 2005.

${ }^{5}$ Kratz, H., et.al., ”Design and Modeling of an Integrated Communications and Thermal Management Module for Microsystem-based Spacecraft” AIAA, J. Spacecraft and Rocket, Submitted paper, October 2005.

${ }^{6}$ Kratz, H., et.al., "Micromachined S-band Patch Antenna with Reduced Dielectric Constant" Sensors and Actuators A: Physical, accepted paper, 2005.

${ }^{7}$ Williams, K.L., et.al., "Electrothermal Feasibility of Cabon Microcoil Heater for Cold/Hot Gas Microthruster” J. Micromech. and Microeng., Submitted paper.

${ }^{8}$ Williams, K.L., et.al., ” Electrothermal Characterization of Tungsten-coated Carbon Microcoil for micropropulsion systems" Carbon, Submitted paper.

${ }^{9}$ Bruhn, F., Rathsman, P., and Stenmark, L., ”Nanospace-1: Spacecraft Design using Advanced Modular Architecture,” AIAA, J. Spacecraft and Rocket, Submitted paper, 2005.

${ }^{10}$ Jain, R. K. and Marathe, B. R., "Variation of Nichrome Film Resistance during Deposition,” Thin Solid Films, Volume 14, Issue 1, 1 December 1972, Pages 155-159.

${ }^{11}$ Imam Kazi, H., Wild P. M., Moore T. N. and Sayer M., ” The Electromechanical Behavior of Nichrome (80/20 wt.\%) Film,” Thin Solid Films, Volume 433, Issues 1-2, 2 June 2003, Pages 337-343.

${ }^{12}$ Holister, G. S., “Experimental Stress Analysis - Principles and Methods,” Cambridge University press, 1967, Chap. 2

${ }^{13}$ Campbel, W. R., "Performance Test of Wire Strain Gages: IV- Axial and Transverse Sensitivities," Technical Note No 1042, National Advisory Committee for Aeronautics, Washington, June 1946.

${ }^{14}$ Dally J. W. and Riley W. F., “Experimental Stress Analysis,” $2^{\text {nd }}$ ed., McGraw-Hill Book Company, 1978, Chap. 6

${ }^{15}$ Higson, G. B., ”Resistance Strain Gauges of Low Temperature Sensitivity,” Journal of Scientific Instruments, Vol. 36, April 1959, pp.157-159.

${ }^{16}$ Vishay Electro-Film. "Nichrome Thin Film, Center Taped Resistors,” Document number 61024 [online database], URL: http://www.vishay.com/docs/61024/ctn.pdf [cited 07 June 2005].

${ }^{17}$ Johansson, S., “Micromechanical Properties of Silicon,” PhD. Thesis, Acta Universitatis Upsaliensis. 1988, page 15.

${ }^{18}$ Madou, M., Fundamental of Microfabrication, CRC Press LLC. 1997, Chap. 4. 